\title{
Consideraciones de diseño para intercambiar electrónicamente recursos académicos digitales a nivel interinstitucional
}

\author{
Design Considerations to Electronically Exchange Digital Academic Resources at \\ Interinstitutional Level
}

\section{Considerações de Projeto para Trocar Electronicamente os Recursos Acadêmicos Digitais a Nível Interinstitucional}

\author{
Diego Alejandro Sierra-Alean ${ }^{1}$ \\ Angie Carolina Suárez-Murcia ${ }^{2}$ \\ Julio Barón-Velandia ${ }^{3}$
}

Recibido: mayo de 2019

Aceptado: noviembre de 2019

Para citar este artículo: Sierra-Alean, D. A.; Suárez-Murcia, A. C.; Barón-Velandia, J. (2020). Consideraciones de diseño para intercambiar electrónicamente recursos académicos digitales a nivel interinstitucional. Revista Científica, 37(1), 107-121. Doi: https://doi.org/10.14483/23448350.14861

\section{Resumen}

Para el intercambio o préstamo de recursos educativos a nivel interinstitucional tanto las entidades públicas como privadas cuentan con procedimientos y especificaciones propios, siendo común el largo periodo requerido para la aprobación de un préstamo y la necesidad de trasladarse a dicha institución para realizar la consulta según la disponibilidad de horario definida. El objetivo de este trabajo es presentar un diseño de software que permita la transacción de recursos académicos entre dos entidades educativas, esto con el fin de reducir tiempos en la ejecución del proceso. La implementación del modelo se basa en las etapas propuestas del método científico y el proceso unificado, aplicando especificaciones de negociación electrónica definidas en ebXML, para definir mediante acuerdos y perfiles condiciones de uso del recurso solicitado. Al poner en funcionamiento el modelo de software se acortan los tiempos de la ejecución del proceso de negocio de varios meses a un par de minutos al realizarse todo el intercambio por medios electrónicos, además de la comunicación sin limitaciones geográficas, lo que favorece tanto a estudiantes como a entidades para el intercambio de propiedad intelectual de manera remota.

Palabras clave: EDI, ebXML, institución educativa, intercambio de recursos académicos, negocio electrónico.

\section{Abstract}

For the exchange or loan of educational resources at the inter-institutional level, both public and private entities have their own procedures and specifications, being common in all the long period of time required for the approval of a loan and the need 
to move to said institution to perform the query according to the availability of defined time. The objective of this paper is to present a software design that allows the transaction of academic resources between two educational entities, in order to reduce time in the execution of the process. The implementation of the model is based on the proposed stages of the Scientific Method and the Unified Process, applying electronic negotiation specifications defined in ebXML, to define, through agreements and profiles, conditions of use of the requested resource. By implementing the software model, the execution times of the business process from several months to a couple of minutes are shortened when all the exchange is carried out by electronic means, in addition to communication without geographical limitations, favoring both students and entities for the exchange of intellectual property remotely.

Keywords: EDI, ebXML, educational institution, electronic business exchange of academic resources.

\section{Resumo}

Para o intercâmbio ou empréstimo de recursos educacionais no nível interinstitucional, tanto entidades públicas como privadas têm seus próprios procedimentos e especificações, sendo comuns em todo o longo período de tempo necessário para a aprovação de um empréstimo e a necessidade de se deslocar para a referida instituição para executar a consulta de acordo com a disponibilidade do tempo definido. O objetivo deste trabalho é apresentar um projeto de software que permita a transação de recursos acadêmicos entre duas entidades educacionais, a fim de reduzir o tempo na execução do processo. A implementação do modelo baseia-se nas etapas propostas do Método Científico e do Processo Unificado, aplicando as especificações eletrônicas de negociação definidas no ebXML, para definir, por meio de acordos e perfis, as condições de uso do recurso solicitado. Ao implementar o modelo de software, os tempos de execução do processo de negócios de vários meses a alguns minutos são encurtados quando toda a troca é realizada por meio eletrônico, além da comunicação sem limitações geográficas, favorecendo tanto os alunos quanto as entidades para o intercâmbio de propriedade intelectual remotamente.
Palavras-chaves: EDI, ebXML, instituição de ensino, intercâmbio de recursos acadêmicos, negócios eletrônicos.

\section{Introducción}

La puesta en funcionamiento de la negociación electrónica permite el intercambio de productos, ya sean físicos o virtuales, por medio de modelos, estándares y arquitecturas enfocadas en la relación negocio a negocio o negocio a cliente. Los modelos de negocio electrónico ofrecen diversos beneficios dependiendo de la implementación en la que son utilizados, algunos de estos son: mayor ventaja competitiva, agilidad en las comunicaciones entre actores del negocio, aumento de la eficiencia, acceso a nuevos mercados, mejoras en la gestión de inventarios y distribución de productos (Adebanjo, Tickle, Lin y Bourlakis, 2016). Además de reducir costos en logística, almacenamiento, personal, tiempos de respuesta, tiempos de entrega y menor procesamiento de transacciones económicas entre los involucrados en el negocio (Alharbi, 2016).

A nivel académico, los recursos con los que cuenta una entidad educativa son componentes importantes de la producción intelectual de las mismas, como resultado de sus actividades de investigación o propuestas que mejoran los métodos tradicionales o establecen una innovación a metodologías comúnmente utilizadas. Cuando se realizan intercambios de recursos académicos entre instituciones educativas, sin importar si estas son públicas o privadas, es necesario establecer con anticipación un convenio interinstitucional en el cual se definan las condiciones para la solicitud, aprobación y préstamo de propiedad intelectual con la ayuda de formatos diligenciados en forma física o electrónica.

No obstante, hay que aclarar que las entidades educativas se enfocan en aspectos específicos dentro de las diferentes ramas de formación, lo cual hace necesario realizar intercambios entre entidades para acceder a otros tipos de recursos académicos por parte de los interesados. Pero, dado que 
estas actividades de negocio no son parte de los objetivos misionales de las instituciones educativas, el proceso es tedioso en cuestión de la larga espera para la aprobación de los recursos solicitados y el desplazamiento físico del solicitante hasta la institución para el estudio o revisión. Estos recursos definen productos y servicios, los cuales, de poder accederse de manera fácil y rápida, ayudarían en el avance científico y tecnológico en diversas áreas del conocimiento.

Con el fin de responder la pregunta: ¿cómo reducir el tiempo requerido en la ejecución del proceso de intercambio de recursos académicos entre entidades educativas, de manera que pueda ser realizado de forma remota?, este documento presenta un modelo software para realizar el proceso de negociación e intercambio electrónico de recursos académicos entre instituciones, utilizando estándares internacionales que permitan crear, compartir y consultar las variables del negocio, alojadas en documentos-contrato que especifican los eventos requeridos para realizar las transacciones, sin abordar los mecanismos y protocolos de seguridad requeridos.

\section{Periodo y muestra de la investigación}

La investigación se realiza en Bogotá, Colombia, e incluye las principales universidades públicas de la ciudad y la generalidad de los casos de las universidades privadas. Se consultó al Sistema Nacional de Bibliotecas y los repositorios institucionales en la ciudad con el fin de encontrar los procedimientos necesarios para realizar la negociación de los convenios interinstitucionales para el acceso a recursos académicos.

\section{Fundamentos teóricos}

\section{Negocios electrónicos}

Los negocios electrónicos se concentran en torno a un determinado tema o sector de servicios dentro de una cadena de valor por medio de la cooperación entre empresas, tanto interna como externamente (Brzozowska y Bubel, 2015), ya que permite llevar a cabo una actividad comercial sin restricciones geográficas y de tiempo.

\section{Electronic Data Interchange (EDI)}

Consiste en el intercambio computador a computador en un formato electrónico de documentos comerciales como, por ejemplo, facturas, órdenes de compra, documentos de inventario, etc., realizado entre dos compañías como socios comerciales. Entre los formatos más utilizados actualmente están ANSI, EDIFACT, TRADACOMS y ebXML (OpenText Corp).

\section{Business to Business (B2B)}

Hace referencia a las transacciones comerciales utilizando tecnología para el intercambio de productos, impulsando la creación de servicios electrónicos y agrupaciones de empresas en portales que permiten a los interesados negociar en mejores condiciones. Engloba las relaciones comerciales de bienes y servicios realizadas exclusivamente entre empresas y en las que no interviene el consumidor final (Fernández, 2018).

\section{Estándares de negocio electrónico}

Existen diversos de modelos de negocio electrónico que aplican el modelo B2B para realizar negociaciones, es decir, herramientas que se han creado para asegurar el envío de documentos entre las entidades; entre las de mayor utilización están: RosettaNet, BizTalk y ebXML.

\section{RosettaNet}

RosettaNet crea y aplica estándares abiertos de procesos de comercio electrónico para toda la industria, formando un lenguaje de comercio electrónico común. Se basa en XML y define pautas de mensajes, interfaz de procesos de negocios y 
marcos de implementación para las interacciones entre empresas. Los socios comerciales pueden definir los aspectos de un proceso de negocio por medio de los Partner Interface Process (PIP), permitiendo describir las acciones entre los actores del negocio, conectándose electrónicamente para procesar transacciones y mover información dentro de sus cadenas de suministro (OpenText Corp).

La figura 1 muestra el intercambio de documentos comerciales utilizando RosettaNet B2B, donde el socio comercial $A B C$ y el socio comercial $X Y Z$ se conectan a internet por una flecha bidireccional denominada B2B. Cada socio comercial utiliza las siguientes características clave: diccionarios RosettaNet, validación RosettaNet, marco de implementación RosettaNet y comprador PIP3A4. (Oracle Corporation, s.f.)

\section{Biztalk}

BizTalk Server es una arquitectura que utiliza adaptadores para enviar y recibir mensajes, pone en funcionamiento procesos comerciales a través de la orquestación e incluye administración y seguimiento de estas diferentes partes. BizTalk Server también incluye la gestión de socios comerciales para mensajería de B2B, alta disponibilidad para maximizar el tiempo de actividad, una plataforma de desarrollo para crear sus propios componentes, una consola de gestión para administrar los artefactos y para el monitoreo de agregaciones de actividad económica, alertas y perfiles (Microsoft Corporation, 2017).

En la figura 2 se muestra un caso sencillo para el intercambio B2B con Biztalk, en la que la organización que realiza el pedido (Purchasing Organization), situada en la parte superior de la figura, ejecuta una orquestación de BizTalk Server que interactúa con dos organizaciones proveedoras. El proveedor A (Supplier A) también utiliza BizTalk Server, con lo que proporciona acceso indirecto a su aplicación de suministro. El proveedor B (Supplier B) usa una plataforma de integración de otro fabricante y se conecta a la orquestación de BizTalk Server de la organización que realiza el pedido por medio de servicios web (Microsoft Corporation, 2018).

$e b X M L$

ebXML son las siglas para Electronic Business Extensible Markup Language. Se trata de un estándar global para la realización de negocios sobre internet establecido bajo las normas ISO en el 2001, con el fin de eliminar documentos escritos en físico, reducir los costos y aprovechar la eficiencia de los productos EDI. Al estar basado en estándares de internet como HTTP, TCP/IP, MIME, SMTP, FTP, UML y XML, puede ejecutarse e implementarse en prácticamente cualquier plataforma

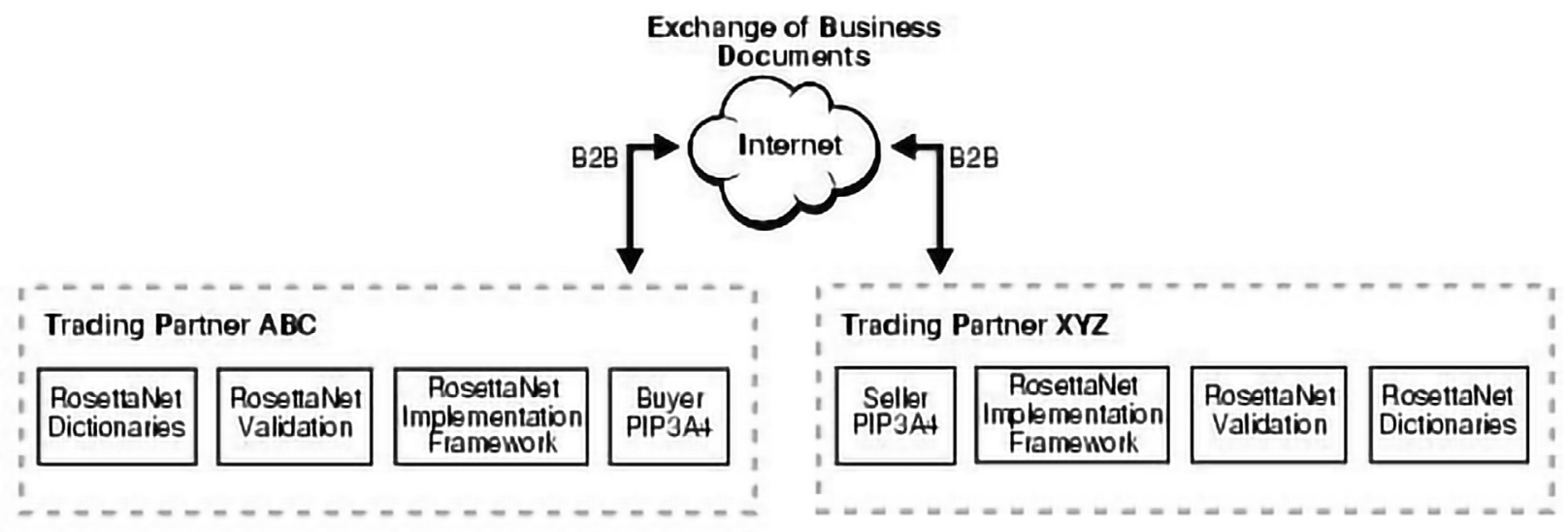

Figura 1. Intercambio de documentos comerciales utilizando RosettaNet B2B. Fuente: Oracle Corporation (s.f.). 


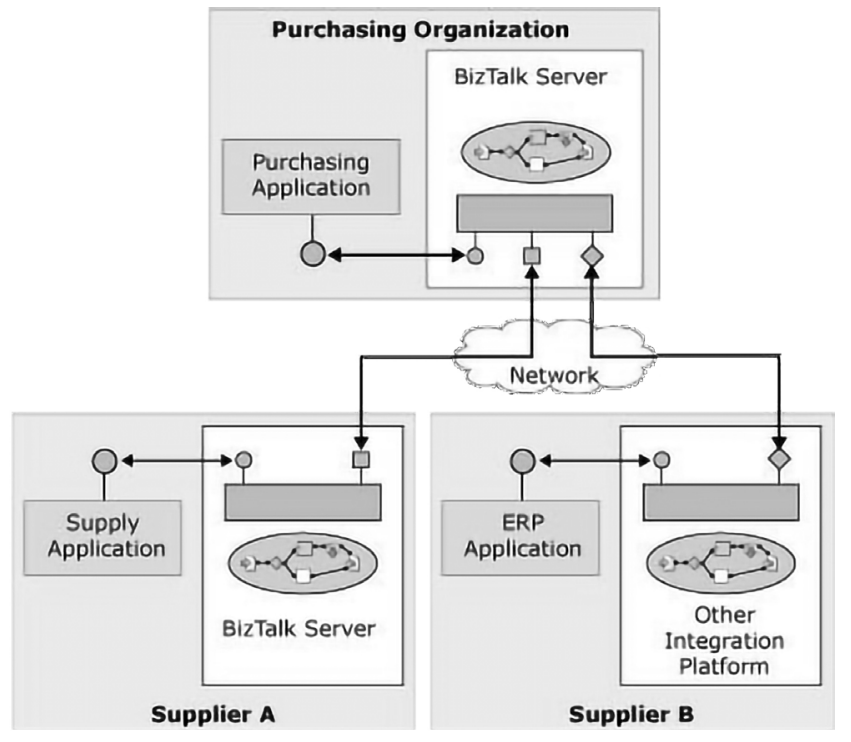

Figura 2. Intercambio de documentos comerciales utilizando Biztalk B2B. Fuente: Microsoft Corporation (2018).

informática. Estas especificaciones permiten definir documentos, actores y elementos que van a interactuar, junto con las funciones que cumple cada uno (OASIS). Es una iniciativa conjunta desarrollada por UN/CEFACT y OASIS (TutorialsPoint).

En el ejemplo del funcionamiento de ebXML con B2B, la compañía A crea o actualiza un perfil de protocolo de colaboración (CPP), el cual es un documento que describe las capacidades, las restricciones y la información técnica del proceso empresarial, para después registrarlo en el ebXML Registry y ponerlo a disposición de sus socios comerciales. La compañía B ingresa al registro en busca de nuevos socios comerciales y descarga el CPP de la compañía A que sea acorde al suyo. Las dos compañías deben llegar a un acuerdo sobre cómo hacer negocios, lo que se denomina acuerdo de protocolo de colaboración (CPA), que se compone de la información de los dos CPP. En este escenario, la compañía B se comunica directamente con la compañía A y envía el CPA recién creado para su aceptación a la empresa $A$. Una vez que la empresa A ha aceptado el CPA, ambas compañías están listas para el negocio electrónico. Este proceso se puede observar en la figura 3 (TutorialsPoint).

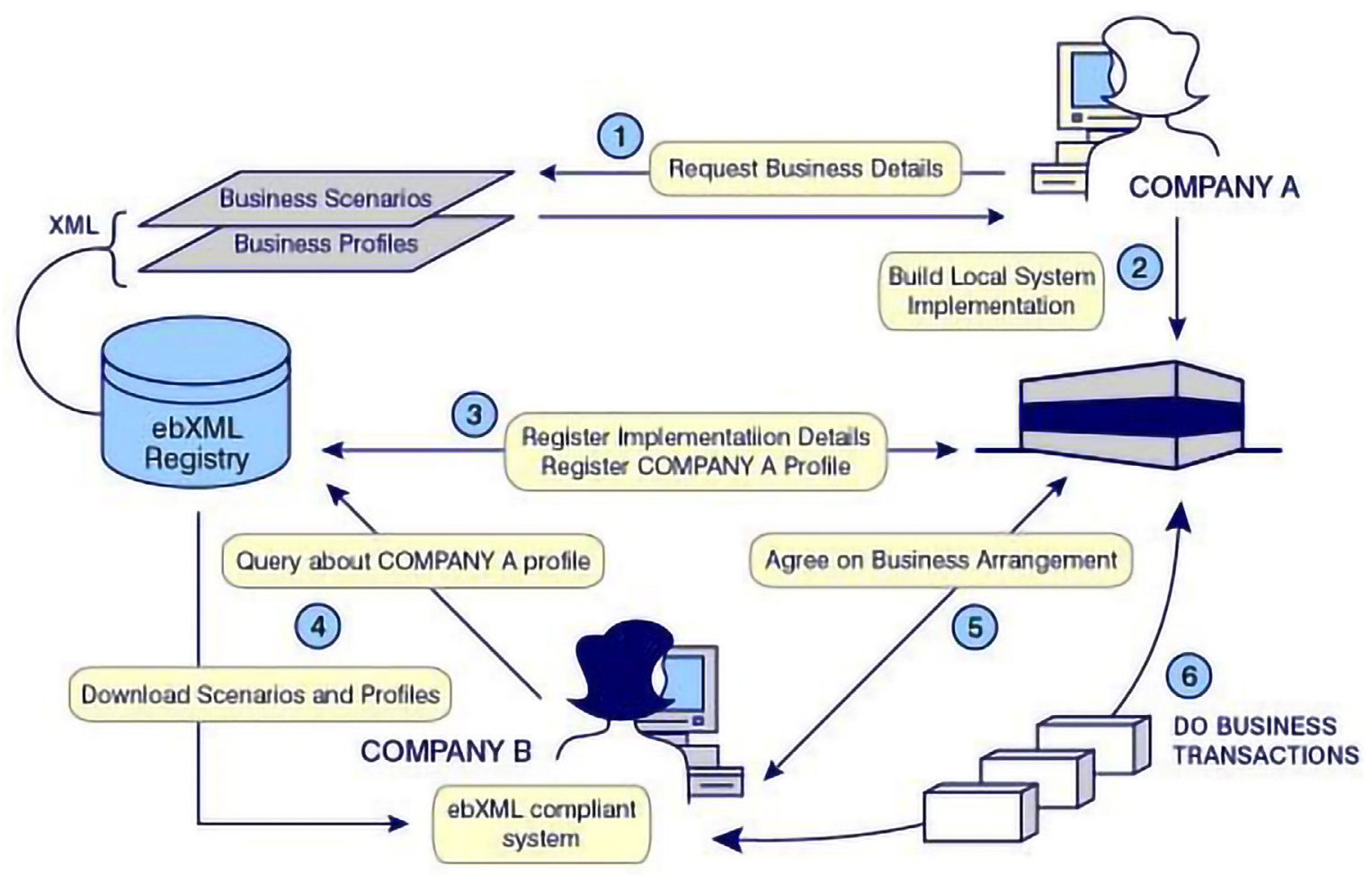

Figura 3. Intercambio de documentos comerciales utilizando ebXML B2B. Fuente: IBM (2001). 
Conceptos fundamentales

Para la realización del modelo de software planteado se requiere comprender los elementos necesarios para la ejecución de una negociación electrónica:

- Gestor de perfiles: divido en dos subcomponentes: el primero se encarga de la interpretación de etiquetas $X M L$, las cuales identifican la información de los perfiles de usuario y los validan mediante formularios web; el segundo subcomponente es el creador de perfiles de usuario que generara el perfil para la institución.

- Generador de acuerdos de negocio: documento que se genera automáticamente uniendo los perfiles de las instituciones. La interfaz de usuario permite la edición del documento, la validación y generación de la especificación en XML.

- Módulo de registro: presenta una arquitectura distribuida en tres o más nodos, siendo uno de estos un árbitro que contiene las interfaces principales del registro que incluyen los perfiles de las entidades, acuerdos y documentos de negocio que pueden ser consultados según la configuración de permisos de usuario. Los otros nodos son clientes que pueden consultar, según la necesidad, los documentos del árbitro.

- Módulo de intercambio de servicios interinstitucionales: presenta al usuario el modelo del negocio por medio de interfaces hacia la aplicación.

- Módulo de presentación de resultados de la consulta: presenta los documentos a la institución que solicite el servicio restringiendo la descarga y copia para mantener los derechos intelectuales, verifica acuerdos de negocio y restringe el servicio cuando terminados o interrumpidos.

\section{Materiales y métodos}

\section{Técnica metodológica}

La técnica metodológica de este proyecto se basa en la aplicación del método científico sobre los estándares de negocio electrónico actuales y el proceso unificado de ingeniería de software (UP) para la implementación del modelo.

\section{Método científico}

Comienza con la etapa de observación de procesos realizados para el intercambio de recursos académicos entre entidades educativas representativas de Bogotá, tanto del sector público como privado, para generalizar los aspectos esenciales de un acuerdo; además del estudio de los principales métodos de negocio electrónico que puedan ser utilizados. En la etapa de inducción se seleccionan tres modelos de transacción electrónica y se realiza una identificación clara del problema. Finalmente, en la experimentación, se efectúa el planteamiento de la solución mediante los datos recolectados obteniendo el modelo de transacción electrónica a utilizar y su especificación para el proyecto.

\section{Proceso unificado de ingeniería de software}

La definición del prototipo se compone de cuatro fases: iniciación, elaboración, construcción y transición. Los hitos de cada una de las fases se describen a continuación:

- Iniciación: primero se analiza el procedimiento en cada institución educativa para el préstamo de recursos académicos, identificando características comunes entre entidades para lograr el préstamo y poder realizar el negocio. Se realiza una depuración de los estándares de negocio electrónico, contrastando los elementos encontrados en las instituciones con los beneficios de cada estándar.

- Elaboración: se divide en dos hitos, el primero tiene en cuenta los resultados obtenidos de las primeras dos fases del modelo científico, encontrando los requerimientos funcionales y no funcionales de la aplicación (generar CPP, generar CPA, realizar acuerdos de negocio, etc.), 
además de identificar cuáles son los principales casos de uso. En el segundo hito se definen los componentes que integran el proyecto basado en los modelos de negocio, además de la interacción que tendrá cada uno de ellos con el cliente final. El diseño se evidencia mediante diagramas UML que plasman actividades, funcionalidad, interacción y secuencia que debe tener el prototipo.

- Construcción: contempla dos hitos, en el primero se abordan los principales casos de uso aplicando desarrollo por componentes; en el segundo se efectúa la integración de los componentes, la especificación de las funciones dependientes entre ellos y los detalles gráficos de usuario que permiten acceder a la funcionalidad.

- Transición: se realizan las pruebas y controles al producto final, además de completar las primeras listas de chequeo de las funcionalidades del mismo. Se realiza la documentación del prototipo y se presenta un ejemplo.

\section{Resultados}

\section{Definición del modelo}

El modelo se basa en la aplicación de ebXML como método para el registro de procesos en un servidor único, la especificación de relaciones, el intercambio de mensajes comerciales y la comunicación en términos comunes (Silva, Torrisi y Pantoni, 2016).

En la figura 4 se puede ver el diagrama de secuencia respectivo a la creación de un documento de especificación de proceso dentro del modelo, basándose en los aspectos que ebXML proporciona para describir cómo una organización lleva a cabo su negocio, en el cual el usuario del prototipo se comunica directamente con un gestor del proceso de negocio desde el cual se genera uno o varios procesos de negocios, que puede contener a su vez una o más colaboraciones compuestas por transacciones, en las cuales se encuentran las descripciones de las características del intercambio y el documento para intercambiar. Una especificación de proceso se puede sintetizar como una declaración de los socios, funciones, colaboraciones, coreografías e intercambios de documentos comerciales que conforman un proceso comercial (TutorialsPoint).

\section{Características del estándar de intercambio electrónico}

Aplica el formato XML para los documentos del negocio, lo cual facilita la lectura y escritura, identificando por etiquetas de padres e hijos las características. La identificación del modelo se realiza por medio de perfiles de cada institución educativa y para su aplicación se dividen en tres grupos diferentes que conforman el documento del perfil: información básica de la institución, empaquetado y comentarios. Esto se realiza mediante etiquetas en la plataforma de la entidad educativa correspondiente, con el fin de que el usuario pueda ingresar los datos básicos de manera sencilla y secuencial a lo largo del proceso de creación de un perfil, tal y como se puede ver en la figura 5 .

El perfil es la presentación y servicios que tendrá cada institución para poder realizar negocios electrónicos, permite que otras entidades puedan consultar y realizar propuestas de acuerdos. Una institución educativa puede tener varios perfiles con diversas funciones a cumplir, y contar con diferentes perfiles para cada rol, esto brinda alternativas para realizar un acuerdo y amplía las posibles solicitudes desacoplando las dependencias de tecnología.

El cumplimiento de la característica de contratación se realiza por medio del acuerdo, el cual es un documento-contrato que incluye: perfiles de las instituciones y parámetros necesarios para validar el negocio. El manejo se realiza por medio de estados del contrato (propuesto, aceptado, activo e inactivo). Las características de seguridad y de transporte de mensajes son incluidas dentro de los perfiles y acuerdos en secciones especialmente dedicadas a ellos. 


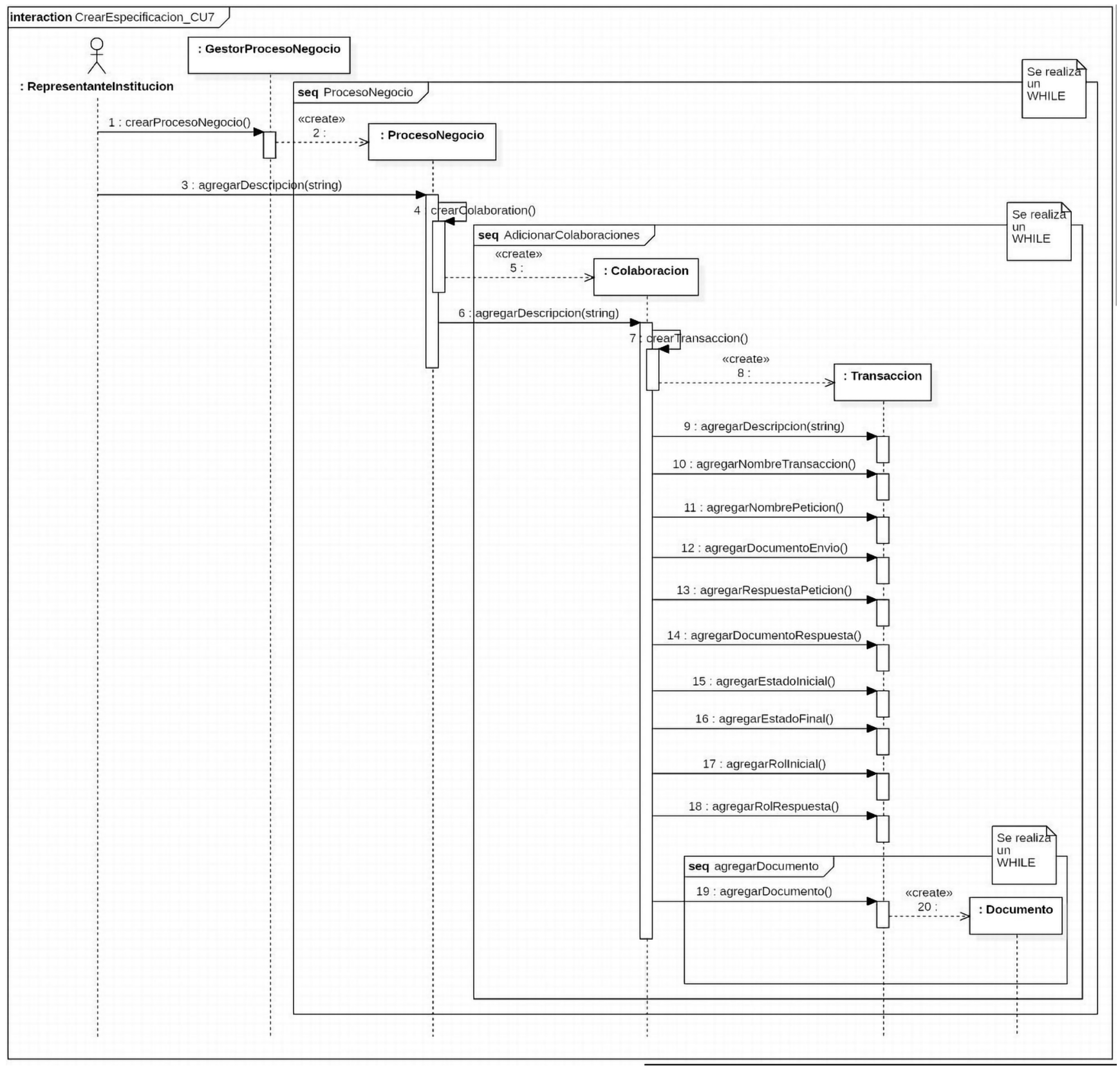

Figura 4. Diagrama de Secuencia para crear una especificación de proceso.
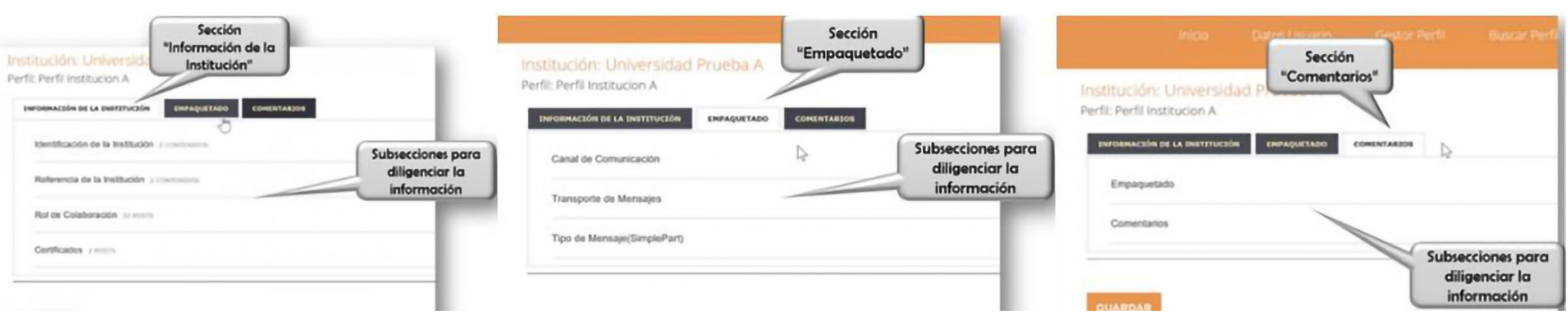

Figura 5. Interfaz gráfica para la creación de un perfil.

Revista Científica •ISSN 0124-2253・e-ISSN 2344-2350 • enero-abril • Bogotá-Colombia • N. 37(1) • pp. 107-121 
Por último, la definición del árbitro se realiza mediante el servidor de registro el cual se encarga de verificar a través de servicios: la comunicación a cada institución educativa y las transacciones realizadas, así como de guardar, mostrar y cifrar los documentos del negocio.

\section{Especificaciones del proceso de intercambio}

Para efectuar el proceso de intercambio, el presente modelo plantea un negocio entre instituciones en el cual se especifican los detalles técnicos, alcance y eventos necesarios. El primer elemento que se debe definir en el proceso de intercambio es el árbitro, encargado de guardar los documentos relacionados a los acuerdos y transacciones, realizar la validación y aprobación de usuarios y contener una base única de estudiantes de todas las entidades educativas registradas. El árbitro es independiente de las instituciones que utilicen el modelo y permite a todos los actores la consulta de los documentos asociados a sus acuerdos.

Para realizar la transacción, cada institución educativa debe registrar una única identificación que lo defina legalmente. El documento del perfil se formaliza mediante reuniones internas en las que se precisan las características aceptadas en los contratos que pueda realizar. Una vez aprobada la especificación de las características y condiciones para la ejecución de contratos, la institución efectúa el registro en el árbitro mediante un CPP para cada escenario.

Para poder aplicar el CPP la institución educativa debe contar con un repositorio en el cual se encuentren los recursos académicos, acorde a los protocolos y detalles presentados en el perfil. Esta implementación se comunica con el servidor árbitro para las transacciones de negocio. En el momento que se encuentre el CPP compatible, se procede a realizar una petición de negocio, la cual se compone de dos partes: acuerdo y especificación de negocio.

La especificación de negocio, como segundo componente, está constituido por un conjunto de documentos que detallan los eventos del proceso de transacción. Cada evento debe ser definido por un documento llamado especificación de proceso que se compone de tres grupos de etiqueta: documento de transacción, transacción de negocio y colaboración binaria.

Después de que la institución realice la propuesta, la contraparte recibirá una notificación del negocio y tendrá que evaluar y responder con la aprobación, modificación o rechazo. En el caso de aprobación, el negocio entra en vigencia dentro de las fechas estipuladas en el acuerdo y ninguna institución puede modificar las condiciones definidas dentro de los términos del negocio. El integrante de una institución de un acuerdo activo se registra en el árbitro con validación previa de la entidad a la cual pertenece; una vez registrado, podrá realizar transacciones, consultar los recursos académicos y los acuerdos vigentes de la entidad en la que está inscrito.

\section{Características del procedimiento de intercambio}

Dentro del procedimiento de intercambio es importante primero definir los parámetros que son necesarios para cumplir el modelo de B2B. En primer lugar, la identificación debe contener la información principal de la entidad y la función que cumple durante la transacción, se utiliza para conformar un contrato legal a través de acuerdos electrónicos. Este contrato es un documento que contiene las fechas de validez del acuerdo, el número de transacciones que se realizarán por acuerdo o límite de invocación, el número de transacciones que podrán realizar los estudiantes en el mismo momento o transacciones concurrentes y los comentarios que describan elementos que acoten el documento.

La definición de los eventos, la seguridad de los mensajes y los canales de comunicación estos son acciones que realizan las partes para poder completar una transacción.

Cada institución debe informar los eventos realizados dentro de su implementación y solicitar la verificación de los contratos. Las interfaces de 
comunicación proporcionadas por la implementación de cada institución, deben cumplir los siguientes servicios:

- Consulta de usuarios registrados de otras instituciones al árbitro.

- Consulta de contratos activos con otras instituciones educativas.

- Registro de transacciones realizadas por usuarios de otras instituciones educativas.

- Consulta de validez de acuerdos.

En la figura 6 se aprecian dos ejemplos de páginas de acceso a dos universidades de prueba.

\section{Diseño}

En esta sección se realiza la descripción del diseño y desarrollo del prototipo.

\section{Actores}

Los actores principales que tendrán acceso a los servicios provistos por el sistema son: el representante de la institución educativa y el estudiante; teniendo en cuenta que los servicios comunes se generalizan en un tipo de actor denominado usuario.

\section{Diagrama de casos de uso}

El modelo posee dos actores principales que interactúan con 16 escenarios del sistema, como se aprecia en la figura 7. Para actualizar un perfil se debe tener en cuenta que este ya debe estar registrado por la entidad, al mismo tiempo que cada perfil puede ser consultado por cualquiera de los actores; en el caso de consultar un acuerdo, cada entidad tiene que haber creado tanto el acuerdo como la especificación de proceso necesaria para dicho acuerdo. Además, se debe realizar la aclaración de la diferencia entre las palabras: recurso y documento. El recurso es el material que va a ser prestado e intercambiado durante el proceso, mientras que el documento se refiere al XML resultante del acuerdo que contiene todos los detalles de la especificación de proceso, incluyendo los CPP de cada universidad.

\section{Diagrama de clases}

El modelo se agrupa en paquetes por funcionalidad, incluyendo clases, relaciones y paquetes, como se observa en la figura 8 . El diagrama de clases cuenta con:

- Modelos: paquete en el cual se encuentran las operaciones (guardar, cargar, consultar datos y objetos) que se comunican con la base de datos.

- Controladores: efectúa operaciones sobre los objetos de la aplicación y su interacción entre ellos. Se encargan de cargar los modelos, las vistas de la aplicación y de enviar mensajes a cada una de ellas.
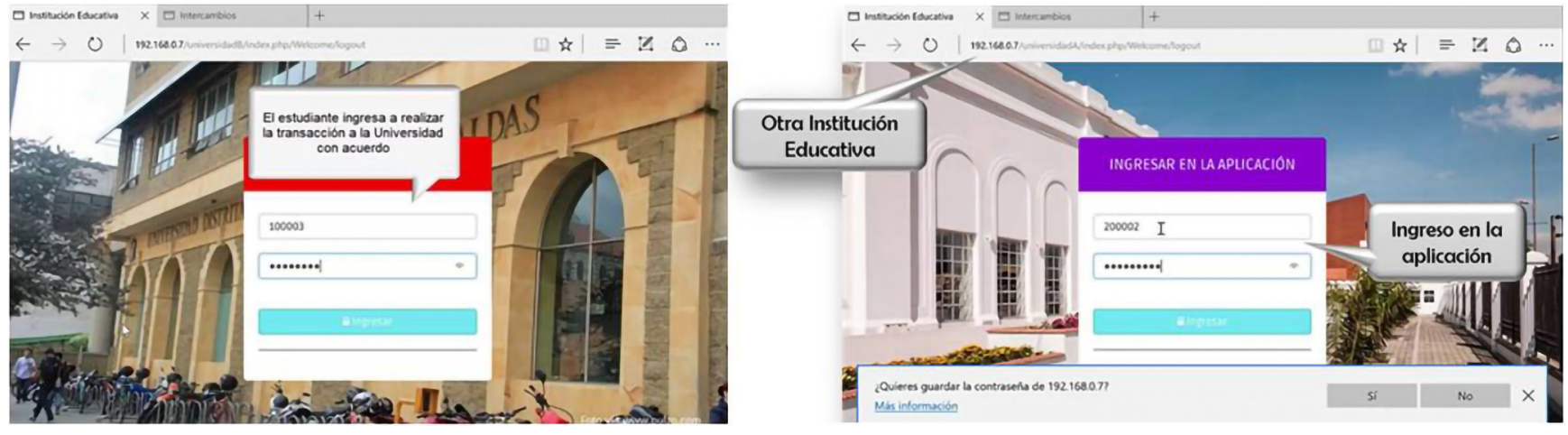

Figura 6. Implementación de plataforma por dos universidades diferentes. 


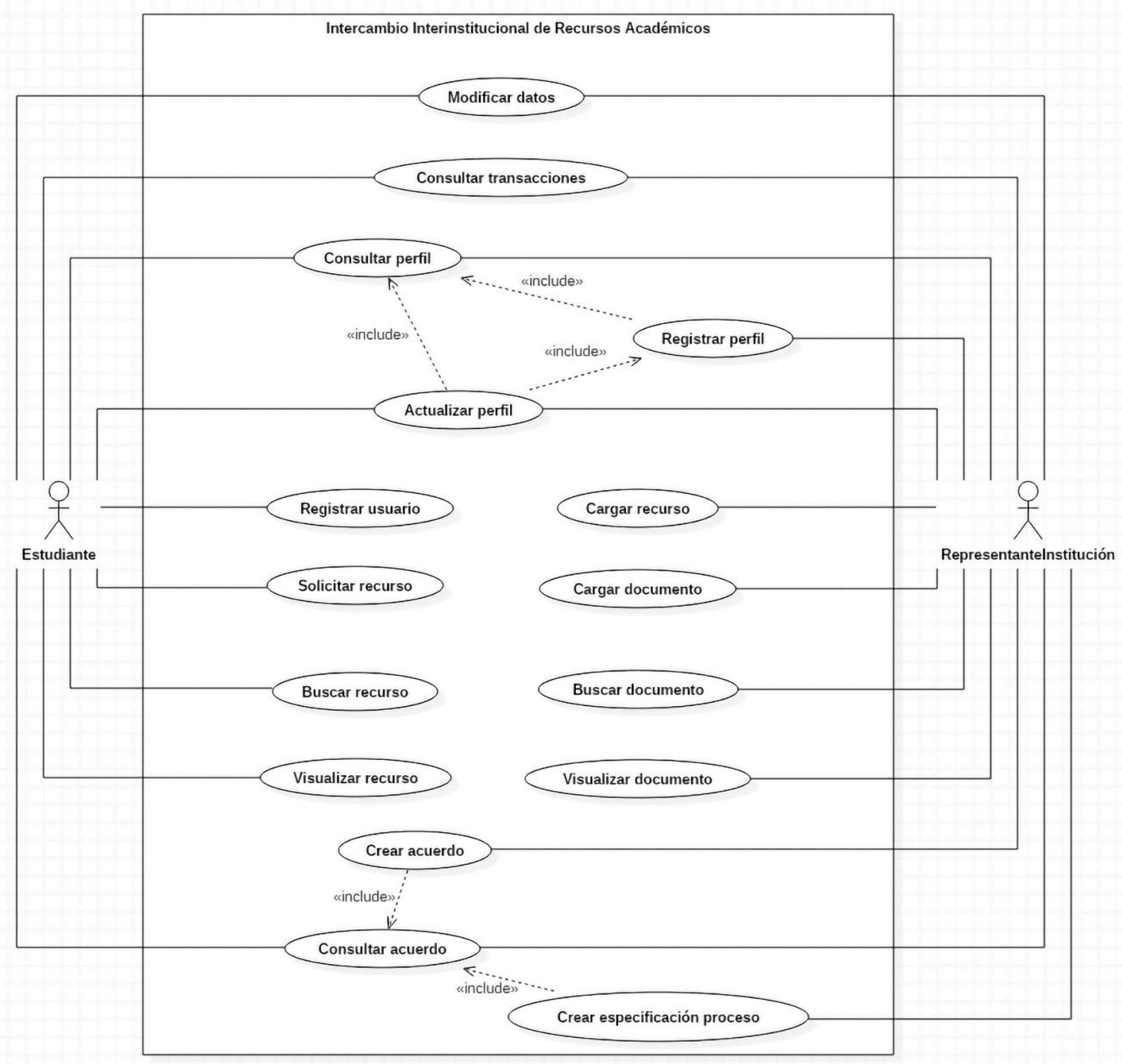

Figura 7. Diagrama de caso de uso intercambio interinstitucional de recursos académicos.

- Usuario: contiene el conjunto de objetos de acceso de datos relacionado con la información del usuario.

- Negocio: representa los objetos de acceso de datos de la gestión de intercambios; contiene: los acuerdos, las especificaciones de proceso, las transacciones de los usuarios, el registro de transacciones realizadas por cada acuerdo de cada entidad y el subpaquete de proceso, el cual permite establecer la unidad mínima de información, contenida en una colaboración y gestionada desde un proceso de negocio.
- Acceso: contiene los objetos de acceso de datos que proporcionan la seguridad del prototipo, su definición de página y los permisos que posee cada usuario para acceder a los contenidos.

\section{Diagrama de despliegue}

Presenta la configuración de los elementos hardware y software según los componentes que lo integran, además de las interfaces que permiten la comunicación entre ellos. Está conformado por 


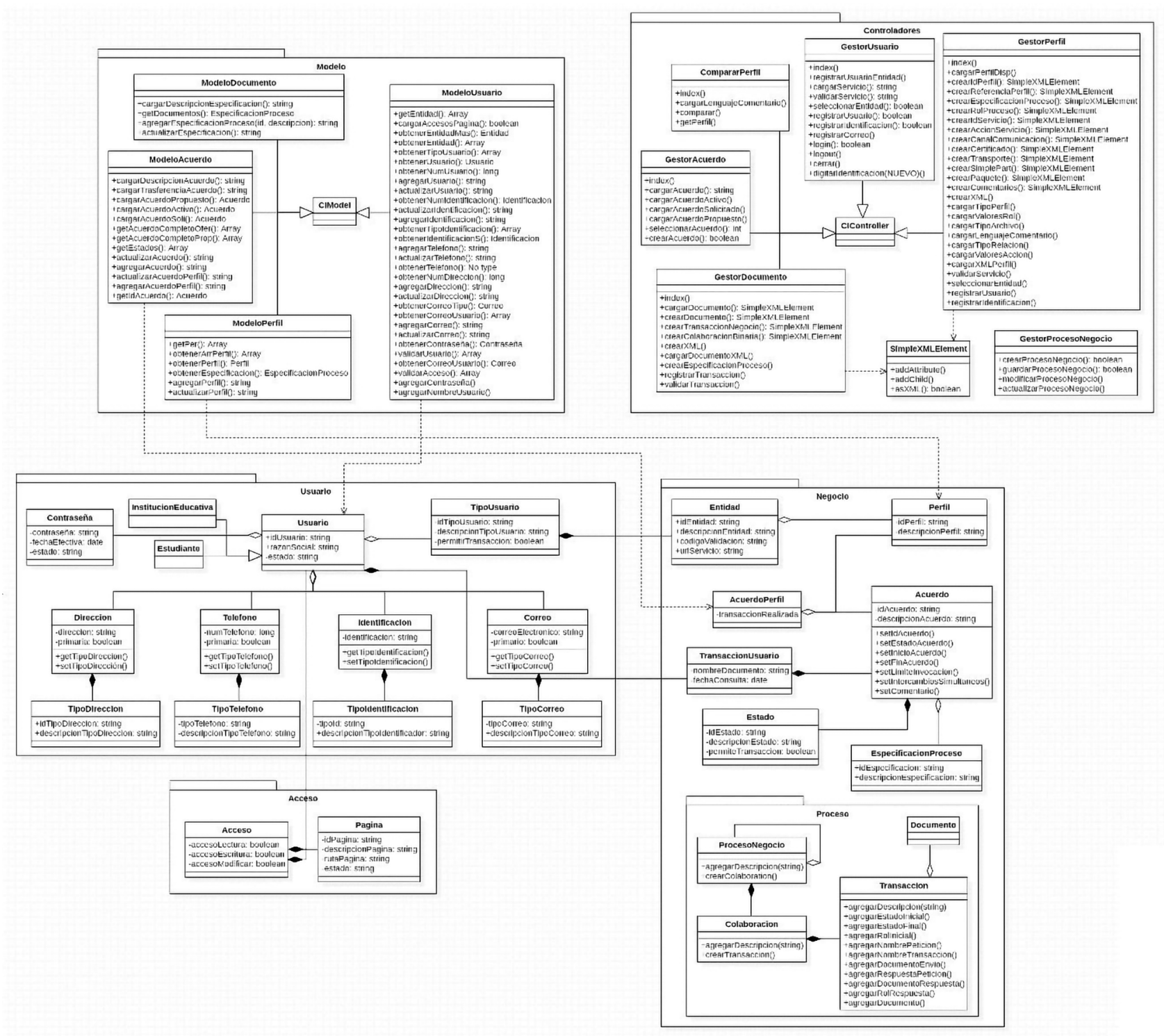

Figura 8. Diagrama de clases del modelo.

cuatro nodos: computador usuario, servidor institución, servidor registro y servidor base de datos, conectados entre ellos por medio de redes TCP/ IP y Ethernet. Cada uno de estos nodos está conformado por componentes que se comunican por medio de interfaces y relaciones de dependencia, como se muestra en la figura 9.

\section{Resultados}

El modelo presentado basa su estructura en un perfil público que identifica de manera explícita los datos básicos y describe las características de infraestructura disponibles de la institución educativa, permitiendo ubicar entidades compatibles a través de un servidor de registro único para realizar propuestas de acuerdo y reducir tiempos en la ejecución, ya que después de que los actores realizan los acuerdos pueden ejecutarse a mayor velocidad al estar apoyados con módulos de software interconectados por protocolos de telecomunicaciones, tales como TCP/IP y Ethernet.

El servidor de registro único se conoce como el servido árbitro, en este las propuestas de negocio 


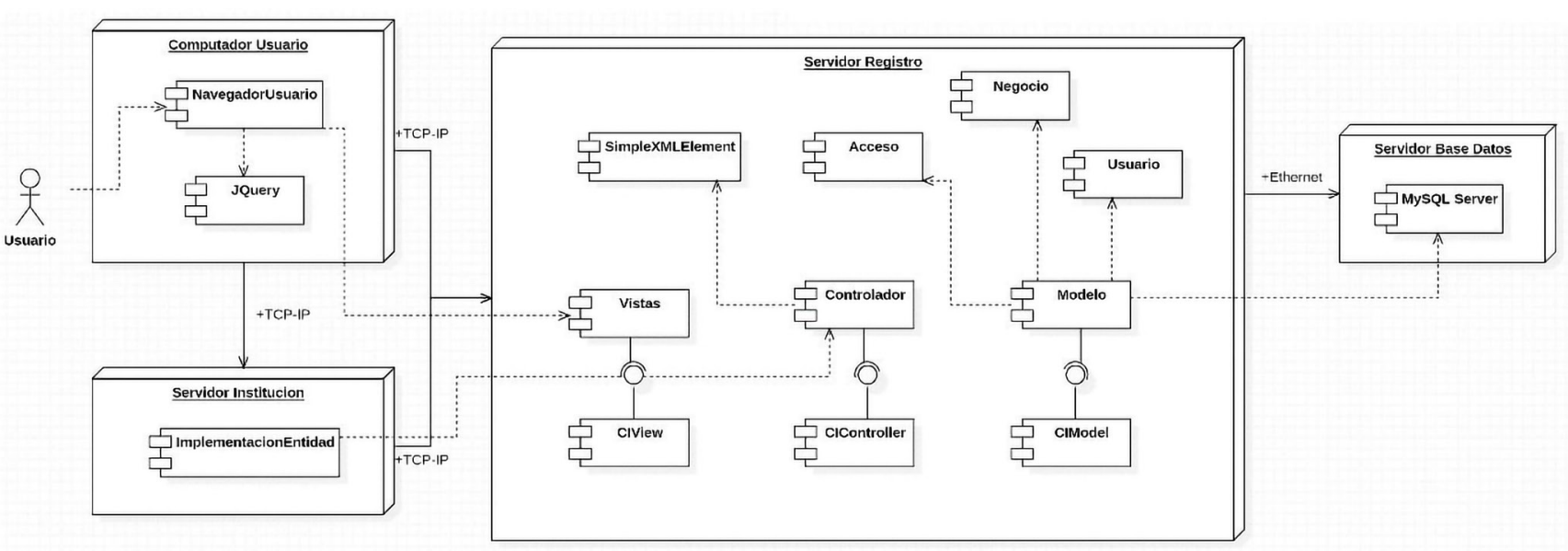

Figura 9. Diagrama de Despliegue del modelo.

y sus cambios se efectúan directamente con él, por medio de mensajes y documentos-contrato que pueden ser consultados por los actores, lo que reduce la necesidad de reuniones presenciales y permite la realización de acuerdos con entidades que pueden estar en lugares distanciados, sin necesidad de traslado de personal. Además, se definen los parámetros generales para la elaboración de la negociación estableciendo un esquema en el cual las diferencias están centradas en los eventos y detalles transaccionales que harán parte del acuerdo.

En cuanto a la definición detallada de los perfiles, es recomendable poner en funcionamiento una estructura independiente de la plataforma de manera individual por cada entidad, para poder especificar dentro de cada acuerdo y convenio interinstitucional los tipos de recursos académicos que pueden ser intercambiados.

\section{Conclusiones}

Para obtener el prototipo propuesto se realizó una investigación sobre los principales modelos de negocio implementados por la industria internacionalmente soportados en especificaciones y pautas ampliamente utilizados. El modelo de ebXML, por ser un estándar abierto, demanda menos recursos económicos para su implementación y cumple con las características necesarias para el B2B, lo cual es fundamental para la representación de procesos de negocio en el ámbito educativo, cuando se cuenta con una amplia diversidad de instituciones (tales como bibliotecas, universidades, colegios, registros, etc.), que dada su naturaleza ofrecen formas diferentes de hacer negociación de recursos. Además, presenta ventajas como: la generación de documentos-contrato de forma que se garantiza que los acuerdos se encuentren en términos internacionales de negocio y, por lo tanto, pueden ser utilizados por diversas entidades geográficamente distribuidas, el manejo de etiquetas XML hace flexible el desarrollo de diversas implementaciones de las estructuras del estándar de intercambio electrónico para definir tanto los términos de los acuerdos como los eventos transaccionales y los perfiles de negocio.

El modelo de software del prototipo disminuye la dependencia de agentes humanos durante la ejecución del proceso de intercambio de recursos de carácter académico, ya que los eventos dentro de las transacciones (el pedido, la solicitud, la aprobación y la validación de los acuerdos) son realizados directamente entre las implementaciones de software de cada institución involucrada en la especificación del acuerdo de colaboración de negocio, siendo el servidor árbitro el encargado 
de verificar y asegurar la especificación de eventos acordados en el momento de la negociación, los límites establecidos en el acuerdo y de registrar las transacciones realizadas por los estudiantes de cada entidad.

Teniendo en cuenta que ya se debe contar con el convenio interinstitucional vigente y activo para realizar un proceso de negocio entre dos entidades educativas, el proceso manual de intercambio de recursos académicos implica una serie de actividades presenciales, en las que cada una posee un promedio de tiempo asignado de acuerdo con la información recolectada tanto de universidades públicas como privadas. Estas actividades son: la solicitud del recurso (de uno a cinco días, dependiendo de la búsqueda dentro de los repositorios de la entidad), espera de disponibilidad (de 20 a 30 días hábiles de acuerdo con la velocidad de procesamiento de la solicitud en los conductos regulares) y consulta directamente en el claustro educativo (alrededor de cinco días que varía según el reglamento de préstamo propio de la institución que posee el recurso), lo cual implica tiempos de hasta dos meses para consultar un material de propiedad intelectual de otra institución educativa. El presente modelo reduce los tiempos tanto de la negociación como de la ejecución del intercambio, ya que el proceso se realiza directamente entre el servidor árbitro y la implementación de la institución educativa, ejecutando el intercambio en el periodo de tiempo en que toma realizar una transacción electrónica para atender la solicitud.

\section{Referencias}

Adebanjo, D.; Tickle, M.; Lin, Y.; Bourlakis, M. (2016). E-business capabilities in developed and developing countries: Different or the same? IEEE International Conference on Management of Innovation and Technology (ICMIT), 19-24. https://doi.org/10.1109/ ICMIT.2016.7605001

Alharbi, A. S. (2016). Future of e-business for SMEs in Saudi Arabia. 3rd International Conference on Computing for Sustainable Global Development (INDIACom), 1383-1389.

Brzozowska, A.; Bubel, D. (2015). E-business as a new trend in the economy. Procedia Computer Science, 65, 1095-1104. https://doi.org/10.1016/j.procs.2015.09.043

Fernandez, S. M. (20 de marzo de 2018). Repositorio Institucional: Propuesta de implementación del modelo B2B del comercio electrónico en el proceso de compras de la empresa Laboratorios AC Farma S.A. http://repositorio.uigv. edu.pe/handle/20.500.11818/2387

IBM (1 de junio de 2001). Understanding ebXML. https://www.ibm.com/developerworks/ library/x-ebxml/

Microsoft Corporation (7 de junio de 2017). Getting started with BizTalk Server. https:// docs.microsoft.com/es-es/biztalk/core/ getting-started-with-biztalk-server

Microsoft Corporation (29 de enero de 2018). Introducing BizTalk Server. https:// docs.microsoft.com/es-es/biztalk/core/ introducing-biztalk-server

OASIS (s. f.). ebXML-Enabling A Global Electronic Market. http://www.ebxml.org/

OpenText Corp. (s. f.). EDI Basics: RosettaNet. https://www.edibasics.com/edi-resources/ document-standards/rosettanet/

OpenText Corp. (s. f.). What is EDI (Electronic Data Interchange)? https://www.edibasics.com/ what-is-edi/

Oracle Corporation (s. f.). RosettaNet B2B Protocol Standard. https://isu.ifmo.ru/docs/ IAS904/integrate.904/b12121/b2bstandards. htm\#1005692

Silva, V. E.; Torrisi, N. M.; Pantoni, R. P. (2016). Negotiation and Collaboration Protocol Based on EbXML Intended to Optimize Port Processes. Information Technology: New Generations, 448, 351-363. https://doi. org/10.1007/978-3-319-32467-8 32

TutorialsPoint (s. f.). ebXML-Business Process. https://www.tutorialspoint.com/ebxml/ebxml business process.htm 
TutorialsPoint (s. f.). ebXML-Introduction. https:// www.tutorialspoint.com/ebxml/ebxml introduction.htm
TutorialsPoint (s. f.). ebXML-Usage Example. https://www.tutorialspoint.com/ebxml/ebxml usage example.htm 